
leading to energy up-converted

SUBJECT AREAS:

MOLECULAR

ELECTRONICS

SPINTRONICS

Received

8 August 2014

Accepted

15 December 2014

Published

14 January 2015

Correspondence and requests for materials should be addressed to A.K.P. (a.pandey@uq. edu.au) electroluminescence in singlet fission photovoltaics

Ajay K. Pandey

School of Chemistry and Molecular Biosciences, The University of Queensland, Brisbane, QLD 4072, Australia.

Free charge generation in donor-acceptor (D-A) based organic photovoltaic diodes (OPV) progresses through formation of charge-transfer (CT) and charge-separated (CS) states and excitation decay to the triplet level is considered as a terminal loss. On the other hand a direct excitation decay to the triplet state is beneficial for multiexciton harvesting in singlet fission photovoltaics (SF-PV) and the formation of CT-state is considered as a limiting factor for multiple triplet harvesting. These two extremes when present in a D-A system are expected to provide important insights into the mechanism of free charge generation and spin-character of bimolecular recombination in OPVs. Herein, we present the complete cycle of events linked to spin conversion in the model OPV system of rubrene/ $\mathrm{C}_{60}$. By tracking the spectral evolution of photocurrent generation at short-circuit and close to open-circuit conditions we are able to capture spectral changes to photocurrent that reveal the triplet character of CT-state. Furthermore, we unveil an energy up-conversion effect that sets in as a consequence of triplet population build-up where triplet-triplet annihilation (TTA) process effectively regenerates the singlet excitation. This detailed balance is shown to enable a rare event of photon emission just above the open-circuit voltage $\left(\mathrm{V}_{\mathrm{OC}}\right)$ in $\mathrm{OPV}$.

T he physical process of singlet exciton fission in organic semiconductors is known to produce two low energy triplet excitons per absorbed photon- a property that has been proposed to increase the thermodynamic limit of optical to electrical energy conversion ${ }^{1-3}$. Traditionally, the exploitation of triplet excitons had been instrumental in improving the luminescence efficiency of organic light emitting diodes (OLEDs) making them technologically competitive for the next generation of energy efficient displays and solid state lighting ${ }^{4-7}$. Triplet excitons are expected to play an equally important role in OPVs by virtue of their long-lifetime. As these triplet excitons can diffuse to longer distances over their singlet counterparts they have greater probability of dissociation into free charges ${ }^{8,9}$. For multiple triplet exciton generation via singlet fission to occur the semiconducting material must satisfy the energetic condition of $E(S 1) \geq 2 E(T 1)$, where $S 1$ and $T 1$ are the corresponding energy levels of singlet and triplet levels ${ }^{1,2}$. If harnessed, the multiple triplet excitons have the potential to improve power conversion efficiencies of OPVs ${ }^{10}$. Furthermore, for efficient energy conversion the theory of detailed balance suggests that an ideal light absorbing semiconductor should also double up as good light emitter. Therefore, the occurrence of electroluminescence in photovoltaics has been used as a thermodynamical indicator of the available $\mathrm{V}_{\mathrm{OC}}{ }^{11,12}$. An ideal solar cell is therefore expected to emit photons just above $\mathrm{V}_{\mathrm{OC}}$; a condition hardly achieved in OPVs due to the non-radiative nature of recombination that pertains the organic heterojunctions ${ }^{13,14}$. In OPVs that are generally composed of electron donating and electron accepting constituents the requirement of charge transfer across the heterojunction means significant quenching of the photoluminescence takes place and therefore it is not surprising that most organic donor-acceptor analogues are poor emitters. In this context, the thermodynamic limit of $\mathrm{V}_{\mathrm{OC}}$ has been usually associated with the photon emission mediated by the bimolecular recombination at CT-state ${ }^{15,16}$. In addition, the interplay between singlet and triplet character of the CT-state further restricts energy relaxation through radiative routes in $\mathrm{OPVs}^{17-19}$. Here, we present steady state measurements detailing the evidence of energy interactions between CT- and triplet-states in the model heterojunction OPV system of rubrene- $\mathrm{C}_{60}$. These interactions have been captured by probing the external quantum efficiency (EQE) of photocurrent generation in the reverse and forward bias of device operation. For the first time, we 
present the spectral evolution of photocurrent in operational OPVs that unveil the triplet character of CT states in charge recombination.

Rubrene is among the most studied organic semiconductors owing to its unique optoelectronic properties such as high carrier mobility, energy-up conversion and long exciton-diffusion lengths ${ }^{20-24}$. It is also known that photoexcitation of rubrene results in formation of long lived multiple triplet states created by singlet fission ${ }^{1,24,25}$. The optical energy gap of rubrene is $2.23 \mathrm{eV}$. The triplet state in rubrene is at $1.14 \mathrm{eV}$ above its singlet ground state. Hence the energetic condition required for singlet fission $E(S 1) \geq 2 E(T 1)$ is endoergic at room temperature with $\Delta \mathrm{E}=-0.05 \mathrm{eV}$. Figure la presents the chemical structures and optical absorption spectrum of rubrene and $\mathrm{C}_{60}$. The energy level diagram for rubrene- $\mathrm{C}_{60}$ based $\mathrm{PV}$ cells is shown in Figure 1b. Schematics detailing the chain of physical events leading to triplet state formation and charge generation that are operational in rubrene and rubrene/ $\mathrm{C}_{60}$ heterojunctions are shown in Figure $1 \mathrm{c}$ and $1 \mathrm{~d}$, respectively. Clearly, the rubrene- $\mathrm{C}_{60}$ heterojunction involves formation of additional states identified as CT and CS. The proximity of triplet state of rubrene with CT and CS manifold provides a unique energy exchange window that is helpful in unveiling the spin character of free charge generation and recombination events.
It is clear from the energy landscape shown in Figure 1 that the rubrene- $\mathrm{C}_{60}$ heterojunction offers a range of additional energy exchange mechanisms. For example in rubrene, as a part of singlet excitation is expected to break into two triplets free charge generation can progress via singlet as well as triplet states. For photocurrent generation through triplet state an electron acceptor with electron affinity significantly lower than $\mathrm{T} 1$ of rubrene is desired. If this condition is not met, as is the case with $\mathrm{C}_{60}$, then singlet fission populated triplet excitons will not take part in free charge generation ${ }^{25}$ and charge generation will progress via the traditional singlet route. As the time scale for singlet fission in rubrene and charge transfer to $\mathrm{C}_{60}$ are comparable (picoseconds) the two events can co-exist. However, the triplet state will still be accessible to any electron back transfer event via the CT-state (Step 4, 5 in Fig. 1d) which is expected to further increase the triplet population. If sufficient amount of triplets are present then the annihilation of two triplets may regenerate a singlet through the TTA process (Step 7). For a complete cycle of free charge generation and recombination a photon emission from the TTA generated singlet exciton becomes an important step (Step 9).

Figure $2 \mathrm{a}$ presents the current-voltage $(\mathrm{J}-\mathrm{V})$ characteristics of rubrene- $\mathrm{C}_{60}$ heterojunction measured in the dark and under AM (a)

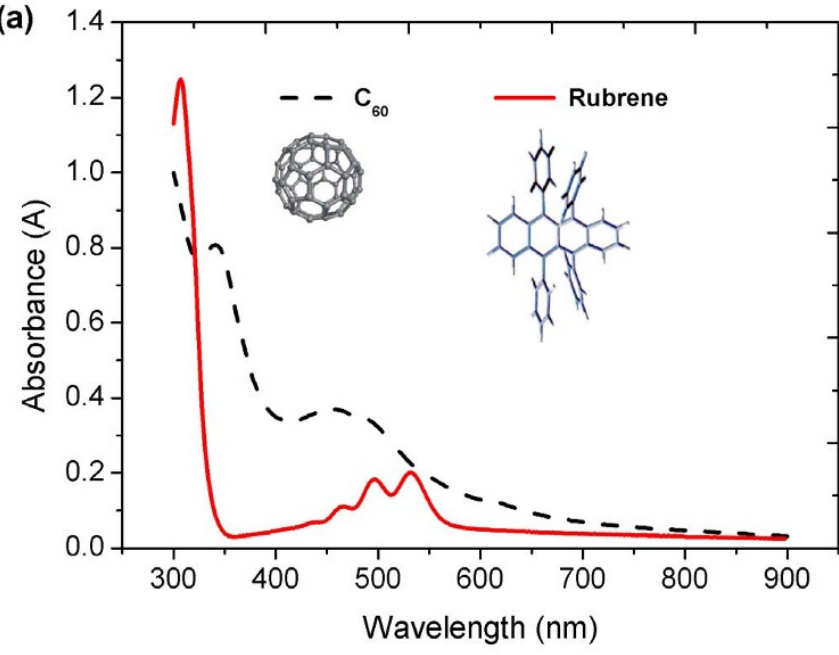

(b)

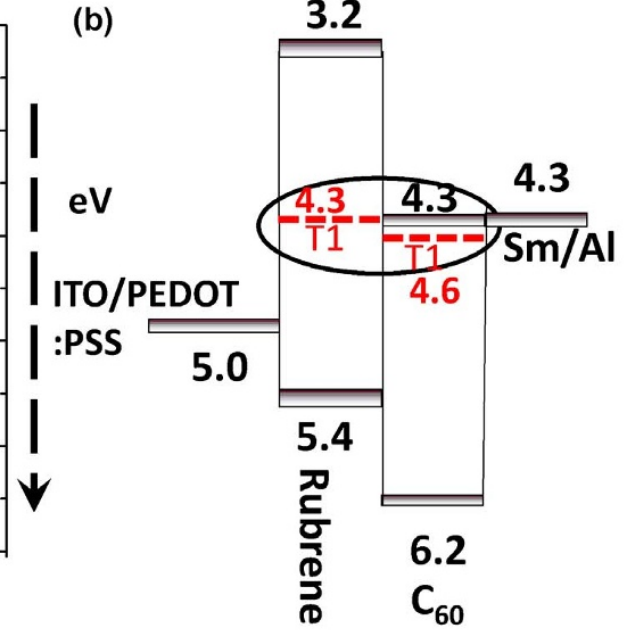

(c)

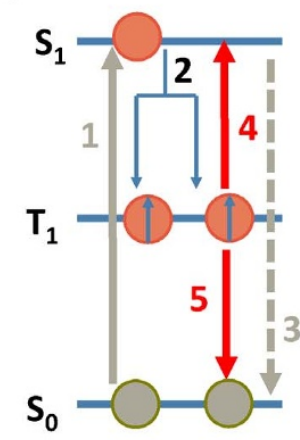

Rubrene (d)

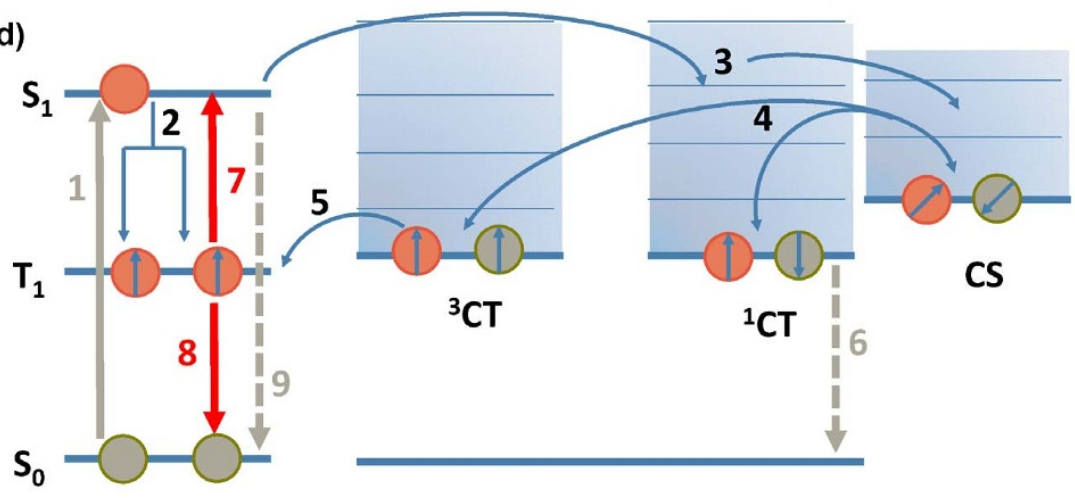

Rubrene/ $\mathrm{C}_{60}$

Figure 1 (a) Optical absorption spectra and chemical structures of Rubrene and $\mathrm{C}_{60}$. (b) Flat band energy alignment showing highest and lowest occupied molecular orbitals (HOMO-LUMO) and location of triplet states at the rubrene- $\mathrm{C}_{60}$ heterojunction. Note that the position of T1 in rubrene $(1.14 \mathrm{eV})$ is very close to the triplet level of $\mathrm{C}_{60}(1.6 \mathrm{eV})$. (c) The detailed cycle of physical events occurring in rubrene thin films (shown as steps $\left.1-5\right)$. A photon absorption event (Step 1) in rubrene is followed by singlet exciton fission (Step 2). Excitation decay to the ground state through two routes - via a prompt emission (Step 3), and delayed emission via triplet-triplet annihilation (Step 4), the non-radiative decay to ground state is also shown (Step 5). (d) In case of rubrene/ $\mathrm{C}_{60}$ - additional processes are: charge transfer/charge separation state formation (Step 3), a possible excitation back transfer from CT state to T1 (Steps 4, and 5). Finally, two triplets can recombine to generate a singlet via TTA (Step 7), which is identified as an energy upconverted emission processes (Step 9). The competing processes to this step would be CT-emission (Step 6) and non-radiative decay to the ground state (Step 8). 

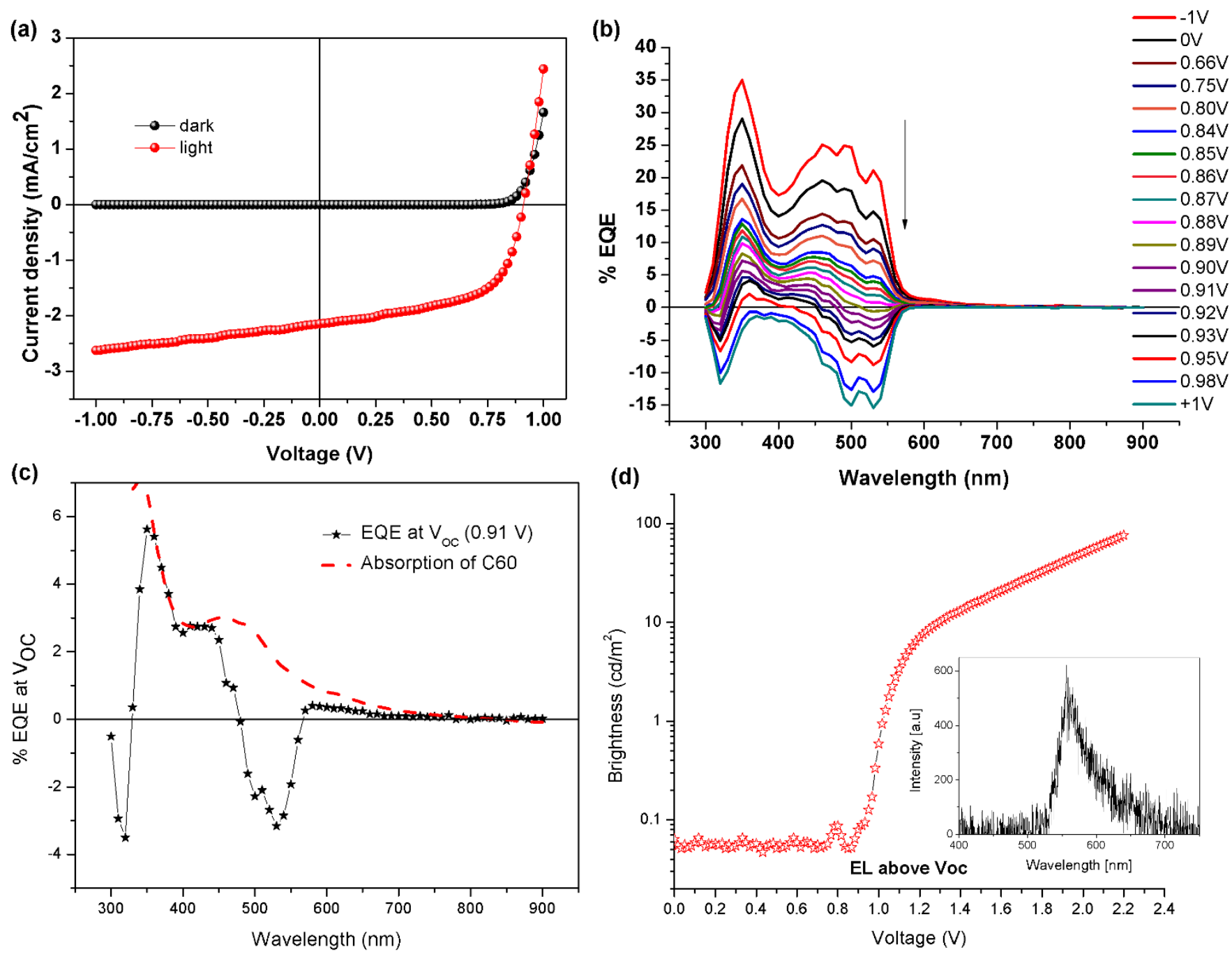

Figure $2 \mid$ (a) Light and dark J-V characteristics of rubrene- $\mathrm{C}_{60}$ heterojunction solar cells. (b) Evolution of photocurrent spectra over the working range of a SF-PV device. The negative component to photocurrent just below the $\mathrm{V}_{\mathrm{OC}}$ is proposed to occur via TTA in rubrene. (c) Comparison of EQE measured at $\mathrm{V}_{\mathrm{OC}}$ with absorption spectrum of thin film of $\mathrm{C}_{60}$. The part positive EQE spectrum matches perfectly with the non-overlapping light absorption region of $\mathrm{C}_{60}$. (d) $\mathrm{L}-\mathrm{V}$ characteristics of rubrene- $\mathrm{C}_{60}$ showing electroluminescence just above $\mathrm{V}_{\mathrm{OC}}$. Here TTA leads to photon emission from the rubrene singlet at a driving voltages $<1 \mathrm{~V}$.

1.5 solar simulator illumination. The heterojunction device structure investigated here is of the following configuration- ITO/30 nm PEDOT:PSS/25 nm Rubrene/35 nm C $60 / 1 \mathrm{~nm} \quad \mathrm{Sm} / 80 \mathrm{~nm} \quad \mathrm{Al}$. Details on rubrene- $\mathrm{C}_{60}$ heteorjunction device fabrication and characterization are presented in Methods. The salient features of device operation are open-circuit voltage; $\mathrm{V}_{\mathrm{OC}}=0.91 \mathrm{~V}$, short-circuit current density; $\mathrm{J}_{\mathrm{SC}}=2.14 \mathrm{~mA} / \mathrm{cm}^{2}$, fill factor; $\mathrm{FF}=0.59$ and power conversion efficiency; PCE $=1.1 \%$. As shown in Figure 1a both rubrene and $\mathrm{C}_{60}$ have strongly absorbing UV peaks featuring at 320 and $350 \mathrm{~nm}$, respectively. The two compounds also absorb significantly in the $450-550 \mathrm{~nm}$ region. Hence under illumination excitons are expected to generate photocurrent via the channel I (photo-induced electron transfer) and II (photo-induced hole transfer) routes ${ }^{26}$. In order to understand the charge generation and recombination interactions via this two routes we present detailed EQE measurements both at short-circuit and in the vicinity of Voc. Figure $2 \mathrm{~b}$ shows the spectral evolution of free charge generation in rubrene- $\mathrm{C}_{60}$ heterojunction cells. At short-circuit $(0 \mathrm{~V})$, the EQE spectrum is identical to the composite absorption of two layers. Here, the high energy peak at $350 \mathrm{~nm}$ is matched to the peak of $\mathrm{C}_{60}$ whereas all three peaks featuring at 460, 490, and $530 \mathrm{~nm}$ are tracked to originate from absorption in the rubrene layer. The small contribution at $620 \mathrm{~nm}$ is matched to the absorption tail of $\mathrm{C}_{60}$. Under application of forward bias the shape of EQE spectrum shows significant changes. The EQE spectra evolve further as the $\mathrm{V}_{\mathrm{OC}}=$ $0.91 \mathrm{~V}$ condition is approached.

It is quite intriguing that the spectral changes in the EQE spectra start to take place at $0.86 \mathrm{~V}$; a value that is still well below the $\mathrm{V}_{\mathrm{OC}}$ $=0.91 \mathrm{~V}$. Here, a part of the photocurrent spectrum is captured to change its sign from positive to negative. We associate this change to triplet exciton generation via bimolecular recombination (Steps 4, 5 of Figure 1d): a process recently proposed as a terminal loss to OPVs by Rao et al. ${ }^{17} \mathrm{~A}$ partly positive and negative photocurrent spectrum below and at $\mathrm{V}_{\mathrm{OC}}$ is indication of the co-existence of photocurrent generation and recombination events in an operational OPV diode. The contribution of the negative part in the EQE spectra becomes stronger at each voltage step approaching the $\mathrm{V}_{\mathrm{OC}}$ condition. As one would expect at $\mathrm{V}_{\mathrm{OC}}$ the positive and negative spectral contributions cancel out and no net photocurrent flows to the external circuit. Going above the $\mathrm{V}_{\mathrm{OC}}$ however, brings a complete change of sign to the EQE spectrum, which is understood to occur as the total photocurrent produced in the forward bias becomes higher than the injected dark current. From dark and light J-V features present above $\mathrm{V}_{\mathrm{OC}}$ in Figure $2 \mathrm{a}$ we can see that this is indeed the case. Figure $2 \mathrm{c}$ 

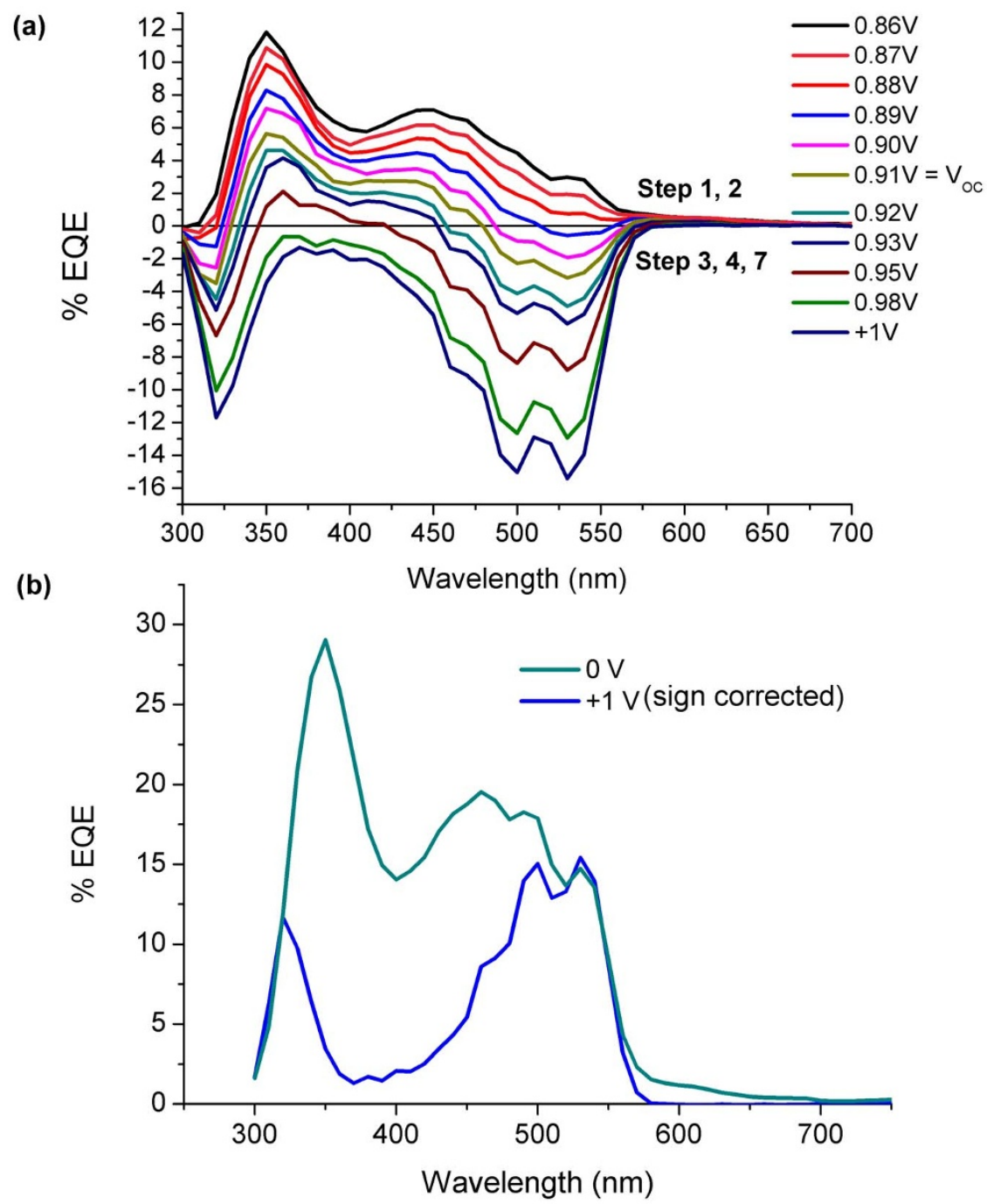

Figure $3 \mid$ (a) The evolution of photocurrent generation from 0.86 V to 1 V. At Voc the charge generation through Step 1 and 2 (of Figure $1 \mathrm{~d}$ ) is shown to interact with TTA linked recombination that is expected to progress via step 3, 4, 7. (b) Comparison of EQE spectra at short-circuit $(0 \mathrm{~V})$ and $+1 \mathrm{~V}$. The spectrum presented for $+1 \mathrm{~V}$ is sign corrected for convenience of comparison. The dominant nature of TTA and direct injection into T1 moves free charge generation entirely to the rubrene layer.

compares the EQE spectrum measured at $\mathrm{V}_{\mathrm{OC}}$ with the thin film absorption spectrum of $\mathrm{C}_{60}$. The positive photocurrent generation is clearly linked to $\mathrm{C}_{60}$ absorption suggesting photocurrent generation via channel II route is not compromised. The negative peaks linked to rubrene suggests that free charge generation via channel I route is being intersected and is now populating the triplet state in rubrene. The favourable energetic condition of $E(S 1) \geq 2 E(T 1)$ makes singlet regeneration via TTA an efficient process. We can expect that regenerated singlet will decay to the ground state by a photon emission event. The luminance vs voltage (L-V) curve shown in Figure $2 \mathrm{~d}$ confirms photon emission takes place just above the $\mathrm{V}_{\mathrm{OC}}$. The electroluminescence spectrum (shown as inset in Figure 2d) measured at $1 \mathrm{~V}$ further confirms the characteristic emission from the rubrene layer ${ }^{20}$. Here, the photon emission at driving voltage of $\sim 1 \mathrm{~V}$ from rubrene is mediated via a TTA led upconversion injection event that is capable of populating singlet state in rubrene. The photon emission event described above at sub-gap driving voltages in rubrene offers an alternative explanation to Auger-type electroluminescence up-conversion previously reported by Pandey and Nunzi ${ }^{21,22,27}$.

Now we turn our attention to the complete absence of $\mathrm{C}_{60}$ exciton signature from the photocurrent spectrum measured in the forward bias. Interestingly, the transition from positive to negative regions in
EQE brings some very noticeable changes to the spectral features associated with photocurrent generation. As can be seen more clearly in Figure $3 \mathrm{a}$, at voltage values marginally above $\mathrm{V}_{\mathrm{OC}}, \mathrm{V}=0.94 \mathrm{~V}$, the positive photocurrent contribution from $\mathrm{C}_{60}$ region gradually vanishes from the EQE spectra. A sign corrected comparison of EQE spectrum recorded at $+1 \mathrm{~V}$ with that recorded at $0 \mathrm{~V}$ in Figure $3 \mathrm{~b}$ shows the associated differences in spectral regions of interest. The EQE spectrum at $1 \mathrm{~V}$ resembles to the neat absorption of rubrene layer shown in Figure 1a, indicating that photocurrent is entirely being generated from rubrene. At operating voltages below the optical gap of rubrene singlet exciton can only be regenerated through the TTA process. For this to progress under external injection at $1 \mathrm{~V}$, one possibility is that the external injection into $\mathrm{C}_{60}$ layer is also progressing via the route taken by back transfer of photogenerated electrons (steps 4, 5 in Figure 1d). This step will result in a triplet population build up in rubrene; a plausible case given the energy proximity of CT-and $\mathrm{T} 1$ - state at rubrene- $\mathrm{C}_{60}$ heterojunction. However, its efficiency will be compromised by a competing CT emission to the ground state (Step 6). For up-converted electroluminescence (UC-EL) to occur efficiently only a steady injection mechanism could sustain the photon emission from rubrene while device is being operated at half of the energy required for injecting an electron into its singlet state. This would mean that electrons injected 


\section{Electrical injection}
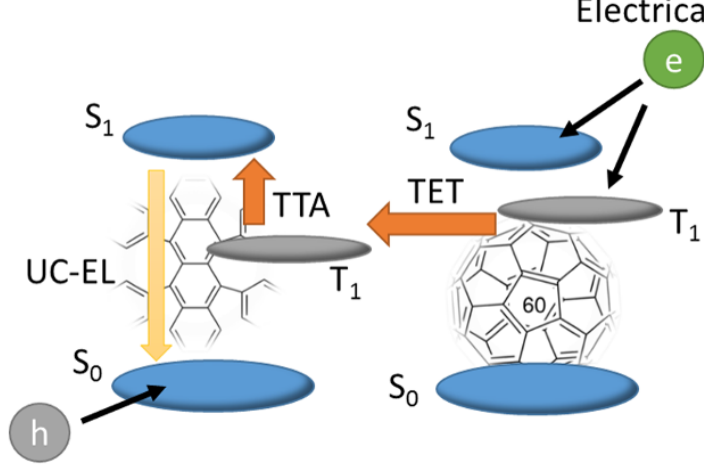

Figure $4 \mid$ The physical model for efficient spin-conversion and energy up-converted electroluminescence at organic heterojunctions. Electrons are injected into $\mathrm{C} 60$ at a spin ratio of $1: 3$. As the $\mathrm{T} 1$ of $\mathrm{C}_{60}$ is higher in energy than $\mathrm{T} 1$ of rubrene a Dexter type triplet energy transfer (TET) from $\mathrm{C}_{60}$ to rubrene is favourable. The TET process then populates triplets in rubrene that undergo triplet-triplet annihilation (TTA) to generate a singlet. The generation of singlet through this route then decays to ground state through UC-EL in rubrene.

into the $\mathrm{C}_{60}$ layer are able to channel through some other routes into rubrene's T1. The obvious question that arises is what makes the UCEL process and associated spin-conversion efficient.

A closer look at the energy level diagram presented in Figure $1 \mathrm{~b}$ suggests that there is a very small energy difference between the LUMO of $\mathrm{C}_{60}$ with respect to its $\mathrm{T} 1$. The $\mathrm{T} 1$ in $\mathrm{C}_{60}$ is reported to be at $\sim 1.6 \mathrm{eV}$ above its ground state ${ }^{28,29}$. The optical gap of $\mathrm{C}_{60}$ and the energy difference between singlet and triplet levels $\left(\Delta \mathrm{E}_{\mathrm{ST}}\right)$ of $\mathrm{C}_{60}$ measured in benzene has been reported to be at $1.99 \mathrm{eV}$ and $390 \mathrm{meV}$, respectively ${ }^{28}$. In Figure 4 we present the schematics of a proposed physical model required for efficient spin conversion and up-converted electroluminescence (UC-EL) in organic heterojunctions. According to spin statistics charge careers are injected into $1: 3$ ratio. The energy alignment of rubrene and $\mathrm{C}_{60}$ is as such that at the application of $+1 \mathrm{~V}$ electrons get injected into $\mathrm{C}_{60}$ and holes in rubrene. As the $\mathrm{T} 1$ of $\mathrm{C}_{60}$ is higher in energy than $\mathrm{T} 1$ of rubrene a Dexter type triplet energy transfer (TET) from $\mathrm{C}_{60}$ to rubrene is favourable. The literature precedence of triplet energy transfer from $\mathrm{C}_{60}$ to rubrene suggests this to be a diffusion-controlled process ${ }^{28}$. The TET process then populates triplets in rubrene that undergo triplet-triplet annihilation (TTA) to generate a singlet. The generation of singlet through this route then decays to ground state in form of an UC-EL event. We note that the heterojunction formation with $\mathrm{C}_{60}$ is essential for efficient spin conversion and up-converted electroluminescence and this phenomenon will be inefficient in neat rubrene based cells that have no $\mathrm{C}_{60}$. Our control measurements confirm this to be the case as in identical device structures of ITO/ $30 \mathrm{~nm}$ PEDOT/25 nm rubrene/1 $\mathrm{nm} \mathrm{Sm} / 80 \mathrm{~nm} \mathrm{Al}$, at least three orders of magnitude lower photocurrent was measured and no upconverted EL was observed. The J-V characteristics, in dark and under illumination, of rubrene only cells are shown in Figure S1. The sustained energy up-converted electroluminescence from rubrene suggests electrons are efficiently converted or injected directly into the triplet state. The sub-gap photon emission provides the direct experimental evidence of delocalized CS-states associated spin mixing at organic heterojunctions under operational conditions.

Next, we discuss the ramifications of spin conversion associated with UC-EL to SF-PVs and OPVs. Essentially, occurrence of UC-EL is a negative event to free charge generation, however mechanisms leading to this behaviour are important for the mechanistic understanding of free charge generation and recombination in OPVs. It is expected that the photon emission mediated via TTA process and one progressing directly from S1 will appear under different injection regimes. At operating voltages below the optical gap of rubrene $(2.2 \mathrm{eV})$ any emission will come from the TTA process. This has significant implications to charge separation and recombination in OPV s and OLEDs. For OPVs, at first, the occurrence of triplet-triplet annihilation induced up-converted electroluminescence event is helpful in tracking the charge recombination trail that is active in OPV devices under operational conditions. As discussed in energetic landscape detailed in Figure 2d, the triplet level in rubrene can be populated via two independent routes- (i) directly via singlet fission, and (ii) via the electron back transfer from the charge transfer state. The heterojunction device energetics (Figure $2 b$ ) are as such that the triplets created by singlet fission do not take part in charge generation but rather serve as a beacon to charge recombination. For the first time we have been successful in capturing the triplet character of charge recombination via ${ }^{3} \mathrm{CT}$ route to $\mathrm{T} 1$ in operational OPVs. As this recombination route is expected to increase the overall triplet population in donor, the voltage point at which sufficient free charges end-up in triplets will impose an energetic limit on the open-circuit voltage creation in OPV systems. These findings show that the ${ }^{3} \mathrm{CT}$ state recombination in organic $\mathrm{D}$-A constituents is a detrimental step in assigning the origin of $\mathrm{V}_{\mathrm{OC}}$ in OPVs as well as SFPVs.

For OLEDs, as will be the case where operating voltages approach and exceed the optical gap, in addition to singlet generated via the TTA process, photon emission will also progress via direct injection into the singlet state of rubrene. The two components have been resolved in rubrene thin films and single crystals by spectroscopic studies where a delayed component (progressing via TTA route with reported PL lifetime $>10 \mathrm{~ns}$ ) is usually associated with the prompt emission (reported PL lifetime $0.2 \mathrm{~ns})^{30,31}$. As emitting species in each case are of the singlet character no change in the emission spectrum of the prompt and delayed signals take place ${ }^{31}$. Clearly, the process of spin-conversion observed in rubrene- $\mathrm{C}_{60}$ offers an alternative route for spin manipulation for light emission at subgap operating voltages in organic semiconductors. A conceptually similar yet fundamentally different approach, based on thermally assisted delayed fluorescence (TADF) in spatially separated donoracceptor moieties, that permits reverse intersystem crossing from T1 to $\mathrm{S} 1$ has been successful in increasing the efficiency of OLEDs ${ }^{32}$.

To summarize, we demonstrated the energy correspondence between CT, CS and triplet state by tracking the steady state photocurrent generation in the model organic heterojunction of rubrene/ $\mathrm{C}_{60}$. The energy proximity of CT, CS and T1 virtually work as an exciton roundabout where excited state enters and exists the rubrene layer by completing a full cycle. The triplet-triplet annihilation effect works as an efficient mechanism for energy up-converted electroluminescence and supports the evidence of formation of a delocalized CS-state at organic heterojunctions. Most importantly, the CT/Cs coupling with T1 opens up a new mechanism for exciton management in triplet states which can be manipulated at will for realization of the next generation of organic optoelectronic and spintronic devices. More importantly, the results are effective in bridging the missing link between our understanding on the nature of spin character of charge injection in organic light emitting diodes (OLEDs) and bimolecular recombination in OPVs.

\section{Methods}

SF-PV Device fabrication and characterization. Sublimed grade rubrene was purchased from Aldrich. High purity $\mathrm{C}_{60}$ was acquired from American Dye Sources. Rubrene- $\mathrm{C}_{60}$ heterojunction devices were grown on clean glass substrates pre-coated with a $150 \mathrm{~nm}$ thick indium tin oxide (ITO) layer with a sheet resistance of $15 \Omega / \square$. A $25 \mathrm{~nm}$ thick Rubrene and $35 \mathrm{~nm}$ thick $\mathrm{C}_{60}$ layers were grown at deposition rate of $0.2-0.3 \mathrm{~A} \%$, in succession without breaking vacuum $\left(10^{-6} \mathrm{mbar}\right)$. For charge collection or confinement a ITO work function modifying layer such as PEDOT:PSS $(40 \mathrm{~nm})$ was used. The PEDOT:PSS was spin coated in air and baked at $180^{\circ} \mathrm{C}$ for $15 \mathrm{~min}$. A $1 \mathrm{~nm} \mathrm{Sm} / 80 \mathrm{~nm} \mathrm{Al}$ was used as top electrode. All devices had an active area of $0.20 \mathrm{~cm}^{2}$. A solar simulator light source (ABET technologies) was used to illuminate devices. All devices were illuminated through the transparent ITO 
electrode at illumination intensity of $100 \mathrm{~mW} / \mathrm{cm}^{2}$. Current-voltage $(\mathrm{J}-\mathrm{V})$ and DCbias dependent EQE measurements were carried out using a modular IV5 I-V testing system and QEX7 - lock-in based EQE testing system from PV Measurements, Inc. The QEX7 system allows sample bias from -1 to $+1 \mathrm{~V}$ in step of $0.1 \mathrm{~V}$. The DCvoltage bias dependent EQE was thus measured at small forward and reverse bias voltages at a chopping frequency of $200 \mathrm{~Hz}$. The QEX7 system automatically subtracts the dark current at each applied voltage and measures the absolute photoresponse. The integration of EQE spectrum over AM 1.5 photon-flux yields photocurrent density that is in excellent agreement with that captured in J-V characteristics. The experimental data presented here is representative of at least 18 devices for each type. The standard deviations in all measured photovoltaic parameters were within $<10 \%$. The current-luminance-voltage measurement was done using an Agilent B1500A semiconductor device analyzer and a calibrated silicon photodiode. The electroluminescence spectra was taken using an Ocean Optics spectrometer.

1. Smith, M. B. \& Michl, Singlet Fission, J. Chem. Rev. 110, 6891 (2010)

2. Chan, W.-L. et al. Observing the multiexciton state in singlet fission and ensuing ultrafast multielectron transfer. Science 334, 154 (2011).

3. Tritsch, J. R. et al. Harvesting singlet fission for solar energy conversion via triplet energy transfer. Nat. Commun. 4, 2679 (2013).

4. Baldo, M. A. et al. Highly efficient phosphorescent emission from organic electroluminescent devices. Nature 395, 151 (1998).

5. Wilson, J. S. et al. Spin-dependent exciton formation in pi-conjugated compounds. Nature 413, 828 (2001).

6. Sun, Y. et al. Management of singlet and triplet excitons for efficient white organic light-emitting devices. Nature 440, 908 (2006).

7. Reineke, S. et al. White organic light-emitting diodes with fluorescent tube efficiency. Nature 459, 234 (2009).

8. Scholes, G. D. \& Rumbles, G. Excitons in nanoscale systems. Nature Mater. 5, 683 (2006).

9. Kohler, A. \& Bassler, H. Triplet states in organic semiconductors. Material Science and Engineering R 66, 71 (2009).

10. Congreve, D. N. et al. External quantum efficiency above $100 \%$ in a singletexciton-fission based organic photovoltaic cell. Science 340, 334-337 (2013).

11. Shockley, W. \& Queisser, H. J. Detailed balance limit of efficiency of p-n junction solar cells. J. Appl. Phys. 32, 510 (1961).

12. Miller, O. D., Yablonovitch, E. \& Kurtz, S. R. Strong internal and external luminescence as solar cells approach the Schokley-Queisser Limit. IEEE J. Photovoltaics 2, 303 (2012).

13. Friend, R. H. et al. Excitons and charges at organic semiconductor heterojunctions. Faraday Discuss. 155, 339 (2012).

14. Lakhwani, G., Rao, A. \& Friend, R. H. Bimolecular recombination in organic photovoltaics. Annu. Rev. Phys. Chem. 65, 557 (2014).

15. Vandewal, K. et al. On the origin of open circuit voltage of polymer-fullerene solar cells. Nat. Mater. 8, 904 (2009).

16. Deibel, C., Strobel, T. \& Dyakonov, V. Role of the charge transfer state in organic donor-acceptor solar cells. Adv. Mater. 22, 4097 (2010).

17. Rao, A. et al. The role of spin in the kinetic control of recombination in organic photovoltaics. Nature 500, 435 (2013).

18. Bakulin, A. A. et al. The role of driving energy and delocalized states for charge separation in organic semiconductors. Science 335, 1340 (2012).

19. Gelinas, S. et al. Ultrafast long-range charge separation in organic semiconductor photovoltaic diodes. Science 343, 512 (2014).

20. Sundar, V. C. et al. Elastomeric transistor stamps: reversible probing of charge transport in organic crystals. Science 303, 1644 (2004).
21. Pandey, A. K. \& Nunzi, J.-M. Rubrene/fullerene heterostructures with a half-gap electroluminescence threshold and large photovoltage. Adv. Mater. 19, 3613 (2007).

22. Pandey, A. K. \& Nunzi, J.-M. Upconversion injection in rubrene/perylenediimide-heterostrucutre electroluminescent diodes. Appl. Phys. Lett. 90, 263508 (2007).

23. Najafov, H. et al. Observation of long-range exciton diffusion in highly ordered organic semiconductors. Nature Mater. 9, 938 (2010).

24. Ryansnyanskiy, A. \& Biaggio, I. Triplet exciton dynamics in rubrene single crystals. Phys. Rev. B 84, 193203 (2011)

25. Reusswig, P. D. et al. Enhanced external quantum efficiency in an organic photovoltaic cell via singlet fission exciton sensitizer. Appl. Phys. Lett. 101, 113304 (2012).

26. Fang, Y. et al. A narrow optical gap small molecule acceptor for organic solar cells. Adv. Energy Mater. 3, 54 (2013).

27. $\mathrm{Ng}, \mathrm{A}$. M. C. et al. Near infrared emission in rubrene:fullerene heterojunction devices. Chem. Phys. Lett. 474, 141 (2009).

28. Arbogast, J. W. et al. Photophysical properties of $C_{60}$. J. Phys. Chem. 95, 11 (1991)

29. Wasielewski, M. R. et al. Triplet States of Fullerenes $\mathrm{C}_{60}$ and $\mathrm{C}_{70}$ : Electron paramagnetic resonance spectra, photophysics, and electronic structures. J. Am. Chem. Soc. 113, 2774 (1991).

30. Ma, L. et al. Singlet fission in rubrene sigle crystal: direct observation by femtosecond pump-probe spectroscopy. Phys. Chem. Chem. Phys. 14, 8307 (2012).

31. Piland, G. B. et al. Magnetic field effects on singlet fission and fluorescence decay dynamics in amorphous rubrene. J. Phys. Chem. C 117, 1224 (2013).

32. Uoyama, H. et al. Highly efficient organic light emitting diodes via delayed fluorescence. Nature 492, 234 (2012).

\section{Acknowledgments}

Author acknowledges the University of Queensland -NSRSG award and Australian Renewable Energy Agency Fellowship (Project 06-F22) for research funding and Dr. Mujeeb Ullah for assistance with electroluminescence measurements. This work was performed in part at the Queensland node of the Australian National Fabrication Facility (ANFF) - a company established under the National Collaborative Research Infrastructure Strategy to provide nano and microfabrication facilities for Australia's researchers.

\section{Additional information}

Supplementary information accompanies this paper at http://www.nature.com/ scientificreports

Competing financial interests: The authors declare no competing financial interests.

How to cite this article: Pandey, A.K. Highly efficient spin-conversion effect leading to energy up-converted electroluminescence in singlet fission photovoltaics. Sci. Rep. 5, 7787; DOI:10.1038/srep07787 (2015).

This work is licensed under a Creative Commons Attribution-NonCommercialNoDerivs 4.0 International License. The images or other third party material in this article are included in the article's Creative Commons license, unless indicated otherwise in the credit line; if the material is not included under the Creative Commons license, users will need to obtain permission from the license holder in order to reproduce the material. To view a copy of this license, visit http:// creativecommons.org/licenses/by-nc-nd/4.0/ 